Piotr Niezgoda ${ }^{1}$, Joanna Sikora², Malwina Barańska ${ }^{3}$, Karolina Obońska ${ }^{3}$, Piotr Adamski ${ }^{1}$, Marek Koziński ${ }^{1}$, Michał Marszałt ${ }^{4}$, Jacek Kubica ${ }^{3}$

${ }^{1}$ Department of Principles of Clinical Medicine, Collegium Medicum, Nicolaus Copernicus University, Bydgoszcz, Poland

2Department of Pharmacology and Therapy, Collegium Medicum, Nicolaus Copernicus University, Bydgoszcz, Poland

${ }^{3}$ Department of Cardiology and Internal Medicine, Collegium Medicum, Nicolaus Copernicus University, Bydgoszcz, Poland

${ }^{4}$ Department of Medicinal Chemistry, Collegium Medicum, Nicolaus Copernicus University, Bydgoszcz, Poland

\title{
Impact of ticagrelor administration strategy on its pharmacokinetics and pharmacodynamics in patients with unstable angina pectoris: a protocol of a randomized study
}

\section{Corresponding author:}

Piotr Niezgoda MD

Department of Principles of Clinical Medicine,

Collegium Medicum,

Nicolaus Copernicus University

9 Sklodowskiej-Curie St.

85-094 Bydgoszcz, Poland

Tel. +48525854023

Fax +48525854024

E-mail: piotr.niezg@gmail.com
Medical Research Journal 2016;

Volume 1, Number 1, 10-14 10.5603/MRJ.2016.0002 Copyright (C) 2016 Via Medica ISSN 2451-2591

\section{ABSTRACT}

Introduction. Dual antiplatelet therapy with aspirin and a P2Y12 receptor inhibitor constitutes an essential part of the management of patients with acute coronary syndromes (ACS). Based on the favorable results of the PLATO trial, ticagrelor is currently recommended as the first line P2Y12 receptor inhibitor in a broad spectrum of ACS patients. According to the recently published data, several conditions, including concurrent analgesia with morphine and clinical presentation as an ACS, may alter ticagrelor absorption and its antiplatelet effect. Therefore, the goal of the present study was to investigate pharmacokinetics and pharmacodynamics of new ticagrelor administration strategies aimed to overcome limitations of the standard ticagrelor loading regimen.

Methods/design. The study is designed as a phase IV, single center, randomized, investigator-initiated, parallel-group, open-label, interventional study comparing the influence of various ticagrelor administration strategies on its pharmacokinetics and pharmacodynamics. Patients with unstable angina pectoris will be randomized in a 1:1:1 ratio into one of three arms, each receiving a $180 \mathrm{mg}$ ticagrelor loading dose (LD). Ticagrelor administration strategies comprise: 1) pulverized ticagrelor administered sublingually, 2) pulverized ticagrelor in $10 \mathrm{~mL}$ suspension in tap water administered orally and 3) integral ticagrelor tablets administered orally. An internal pilot study including 30 (10 in each of the arms) is planned in order to determine the final sample size. The primary endpoint of the trial is time $\left(t_{\max }\right)$ required for ticagrelor and its active metabolite AR-C124900XX to reach maximum plasma concentration within time frame of six hours after administration of ticagrelor LD. The secondary endpoints include ticagrelor and AR-C124900XX maximum plasma concentration, area under the plasma concentration-time curve for ticagrelor and AR-C124900XX (AUC 0-6h) and platelet reactivity assessed with Multiple Electrode Aggregometry using the Multiplate ${ }^{\mathrm{TM}}$ Analyzer prior to and within time frame of six hours following ticagrelor LD.

Discussion. This study is expected to provide essential evidence-based data on the impact of ticagrelor administration strategy on its pharmacokinetics and pharmacodynamics in patients with unstable angina pectoris. Hopefully, based on its results, further clinical outcome-powered trials on new ticagrelor administration strategies will be designed and conducted.

Key words: ticagrelor administration, ACS, pharmacokinetics, pharmacodynamics, angina

Med Res J 2016; 1 (1): 10-14 


\section{Introduction}

Based on the guidelines of the European Society of Cardiology (ESC), antiplatelet therapy comprising aspirin and a P2Y12 receptor inhibitor is a recommended regimen for patients with acute coronary syndromes (ACS) [1, 2]. Clinical advantages of either ticagrelor or prasugrel over clopidogrel have been proven in large clinical trials, such as the TRial to assess Improvement in Therapeutic Outcomes by optimizing platelet inhibitioN with prasugrel-Thrombolysis In Myocardial Infarction 38 (TRITON-TIMI 38) or PLATelet inhibition and patient Outcomes (PLATO) studies, thus making these agents preferable P2Y12 receptor inhibitors in ACS patients [3-9]. Importantly, ticagrelor is currently recommended as the first line P2Y12 receptor inhibitor in a broad spectrum of ACS patients, also in subjects managed conservatively and in patients who are likely to undergo coronary artery bypass surgery, in the subsets where prasugrel should be avoided [1].

It is believed that adequate platelet inhibition is crucial during percutaneous coronary intervention $(\mathrm{PCl})$ and in the periprocedural period, particularly in patients undergoing coronary stenting, because implantation of thrombogenic stent into the thrombotic lesion exposes patients to the risk of stent thrombosis, a potentially fatal complication. Therefore, routine immediate administration of antiplatelet agents, just after making the initial diagnosis, is recommended in all ACS patients with the exception of prasugrel, which should not be given in subjects with non-ST elevation ACS until coronary angiography is completed [1].

Notably, morphine is considered a drug of choice for chest pain alleviation in patients presenting with acute myocardial infarction [2]. Nevertheless, based on the available data, morphine, an opioid analgesic, may lead to decreased clopidogrel plasma concentration and its attenuated antiplatelet action if both drugs are administered simultaneously [10]. Additionally, our recent randomized study indicated that morphine delays and attenuates ticagrelor exposure and action in patients with myocardial infarction [11-13]. Similarly, morphine was also demonstrated to decrease ticagrelor concentrations, but not its antiplatelet effects, in healthy volunteers [14]. Besides morphine co-administration, other clinical conditions (e.g. clinical presentation with an ACS, particularly ST-segment elevation myocardial infarction (STEMI), concomitant cardiogenic shock, unconsciousness, incapability to swallow, malabsorption, therapeutic hypothermia) may reduce absorption of P2Y12 receptor inhibitors and/or their antiplatelet action [15-18].
Interestingly, Zafar et al. demonstrated higher bioavailability of crushed vs. integral clopidogrel tablets in healthy volunteers [19]. Similarly, administration of pulverized vs. integral ticagrelor tablets was associated with increased antiplatelet effect in STEMI patients in the Mashed Or Just Integral pill of TicagrelOr (MOJITO) study [20].

These reports provide a solid rationale for new ticagrelor administration strategies, which may overcome limitations of the standard ticagrelor loading regimen. Thus, we designed a study evaluating differences in ticagrelor pharmacokinetics and pharmacodynamics in patients who received pulverized tablets either orally or sublingually in comparison with conventional oral administration of integral tablets.

\section{Methods}

The trial is designed as a phase IV, single center, randomized, investigator-initiated, parallel-group, open-label, interventional study aimed to evaluate the influence of ticagrelor administration strategies on its pharmacokinetics and pharmacodynamics in patients hospitalized for unstable angina pectoris. The protocol of the study was approved by The Ethics Committee of Nicolaus Copernicus University in Toruń, Ludwik Rydygier Collegium Medicum in Bydgoszcz (approval number KB 540/2015). The study is conducted in compliance with the regulations established in the Declaration of Helsinki. Each participant needs to sign a written informed consent before enrollment into the trial. The eligibility criteria for enrollment into the study include male and non-pregnant female patients in the age range of 18-80 years, diagnosed with unstable angina pectoris whose mortality risk score was assessed $<140$ points according to GRACE Score, who signed a written consent for coronary angiography $\mathrm{PCl}$, if needed. Key exclusion criteria include ongoing (or terminated within 14 preceding days) treatment with any P2Y12 receptor inhibitor, treatment with oral or parenteral anticoagulants, history of intracranial hemorrhage or recent (defined as last 30 days) gastrointestinal hemorrhage, coagulation disorders, severe chronic pulmonary disorders, second or third degree atrioventricular block, Killip class III or IV on the point of screening. The full list of inclusion and exclusion criteria is presented in Table 1.

The study site is The Department of Cardiology, Antoni Jurasz University Hospital in Bydgoszcz, Poland. Patients diagnosed with unstable angina pectoris who signed the informed consent, are subsequently randomized in a 1:1:1 manner into one of three arms each receiving a $180 \mathrm{mg}$ ticagrelor loading dose (LD). Ticagrelor administration strategies comprise: 1) pulverized ticagrelor administered sublingually, 2) pulverized 
Table 1. The complete list of inclusion and exclusion criteria used in the study

\begin{tabular}{|c|c|}
\hline Inclusion criteria & Exclusion criteria \\
\hline $\begin{array}{l}\text { Provision of informed consent prior to any study } \\
\text { specific procedures } \\
\text { Clinical diagnosis of unstable angina } \\
\text { Male or non-pregnant female, aged } 18-80 \\
\text { Provision of informed consent for angiography } \\
\text { and percutaneous coronary intervention (PCl) } \\
\text { GRACE score }<140 \text { pts }\end{array}$ & $\begin{array}{l}\text { Treatment with ticlopidine, clopidogrel, prasugrel or ticagrelor within } \\
14 \text { days before the study enrollment } \\
\text { Hypersensitivity to ticagrelor } \\
\text { Current treatment with oral anticoagulant or chronic therapy with low- } \\
\text {-molecular-weight heparin } \\
\text { Active bleeding } \\
\text { History of intracranial hemorrhage } \\
\text { Recent gastrointestinal bleeding (within } 30 \text { days) } \\
\text { History of coagulation disorders } \\
\text { Platelet count less than }<100 \times 10^{\wedge} 3 / \mathrm{mcL} \\
\text { Hemoglobin concentration less than } 10.0 \text { g/dL } \\
\text { History of moderate or severe hepatic impairment } \\
\text { History of major surgery or severe trauma (within } 3 \text { months) } \\
\text { Patients considered by the investigator to be at risk of bradycardic events } \\
\text { Second or third degree atrioventricular block during screening for eligibility } \\
\text { History of asthma or severe chronic obstructive pulmonary disease } \\
\text { Patient requiring dialysis } \\
\text { Manifest infection or inflammatory state } \\
\text { Killip class III or IV during screening for eligibility } \\
\text { Respiratory failure } \\
\text { History of severe chronic heart failure (NYHA class III or IV) } \\
\text { Concomitant therapy with strong CYP3A inhibitors (ketoconazole, } \\
\text { itraconazole, voriconazole, telithromycin, clarithromycin, nefazadone, } \\
\text { ritonavir, saquinavir, nelfinavir, indinavir, atazanavir) or strong CYP3A } \\
\text { inducers (rifampicin, phenytoin, carbamazepine, dexamethasone, } \\
\text { phenobarbital) within } 14 \text { days and during study treatment } \\
\text { Body weight below } 50 \text { kg }\end{array}$ \\
\hline
\end{tabular}

ticagrelor in $10 \mathrm{~mL}$ suspension in tap water administered orally and 3) integral ticagrelor tablets administered orally. Randomization is conducted using Random Allocation Software version 1.0. Coronary angiography is performed at least six hours after the enrollment into the trial, after completing the blood sample collecting schedule. The study results will be reported in line with the CONSORT statement [21, 22]. The scheme of the study is presented in Figure 1.

\section{Endpoints}

The primary endpoint of the trial is time $\left(t_{\max }\right)$ required for ticagrelor and its active metabolite AR-C124900XX to reach maximum plasma concentration within time frame of six hours after administration of ticagrelor LD. The secondary endpoints include ticagrelor and AR-C124900XX maximum plasma concentration, area under the plasma concentration-time curve for ticagrelor and AR-C124900XX (AUC 0-6h) and platelet reactivity assessed with Multiple Electrode Aggregometry (MEA) using the Multiplate ${ }^{\mathrm{TM}}$ Analyzer prior to and within time frame of six hours following ticagrelor LD.
All the study endpoints together with details regarding sampling are listed in Table 2.

\section{Blood sample collection}

Blood collection using an intravenous catheter is scheduled directly prior to ticagrelor LD and 15, 30, $45,60,120,180,240,360$ minutes following LD. Blood collection is performed by cardiology intensive care nurses and is supervised by the physician responsible for previous eligibility screening for each patient.

\section{Pharmacokinetics and pharmacodynamics}

Pharmacokinetic assessments of all blood samples obtained according to the schedule are performed in The Department of Medicinal Chemistry, Nicolaus Copernicus University, Ludwik Rydygier Collegium Medicum in Bydgoszcz. Concentration of ticagrelor and its active metabolite (AR-C124910XX) are determined with liquid chromatography tandem mass spectrometry. Pharmacodynamic measure- 
ments for the sake of the trial are performed using Multiple Electrode Aggregometry (MEA; the Multiplate ${ }^{\mathrm{TM}}$ Analyzer, Roche Diagnostics International Ltd, Rotkreuz, Switzerland). MEA will be used in all enrolled participants. Pharmacokinetic and pharmacodynamic analyzes are performed by blinded skilled investigators. Both methods have been described in details previously [11, 12, 23-25].

\section{Pilot study}

We plan to perform an internal pilot study including 30 (10 in each of the arms) in order to determine the final sample size.

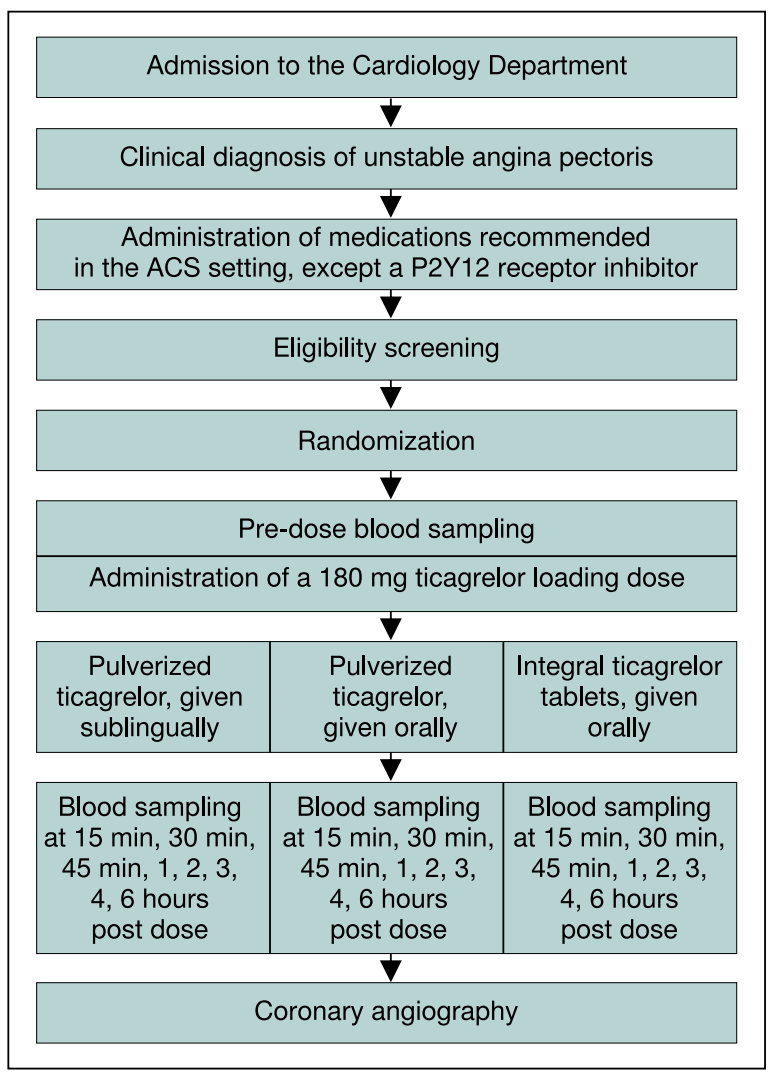

Figure 1. The schematic protocol of the study

\section{Safety of the trial}

The study population is limited only to patients diagnosed with unstable angina pectoris, whose mortality risk is low or intermediate, as estimated by the GRACE score (< 140 points). Moreover, all participants receive medications of all other groups recommended by the ESC guidelines for the ACS management, e.g. aspirin, statins, beta-blockers, angiotensin converting enzyme inhibitors or angiotensin II receptor inhibitors. Every case of patient's condition deterioration leading to the necessity of immediate coronary angiography will result in the discontinuation of that patient's participation in the trial so as to ensure appropriate therapy. For the entire hospitalization period, the study participants will receive ticagrelor maintenance dose of $90 \mathrm{mg}$ twice daily with the strong recommendation to continue therapy with ticagrelor after discharge. Ticagrelor may be replaced with clopidogrel (a $600 \mathrm{mg}$ clopidogrel loading dose will be administered) in patients unable to continue such treatment, mainly due to financial reasons, on the day of discharge from The Department of Cardiology.

\section{Discussion}

This study is expected to provide essential evidence-based data on the impact of ticagrelor administration strategies on its pharmacokinetics and pharmacodynamics in patients with unstable angina pectoris. Hopefully, based on its results, further clinical outcome-powered trials on new ticagrelor administration strategies will be designed and conducted.

\section{The study status}

The study is currently recruiting participants. It was registered in the ClinicalTrials.gov database and received identifier NCT02612116.

Table 2. The list of study endpoints

\section{Primary endpoint of the study Secondary endpoints of the study}

Time to maximum concentration $\left(\mathrm{t}_{\max }\right)$ for ticagrelor and AR-C124900XX [Time frame: 6 hours]
Maximum ticagrelor and AR-C124900XX concentration [Time frame: 6 hours]

Area under the plasma concentration-time curve for ticagrelor (AUC 0-6 h)

[Time frame: pre-dose and $15 \mathrm{~min}, 30 \mathrm{~min}, 45 \mathrm{~min}, 1$, 2, 3, 4, 6 hours post dose]

Area under the plasma concentration-time curve for AR-C124900XX (AUC 0-6h)

[Time frame: pre-dose and $15 \mathrm{~min}, 30 \mathrm{~min}, 45 \mathrm{~min}, 1$, 2, 3, 4, 6 hours post dose]

Platelet reactivity assessed by Multiple Electrode Aggregometry [Time frame: pre-dose and $30 \mathrm{~min}, 1,2,3,4,6$ hours post dose] 


\section{Acknowledgements}

We would like to thank all cardiologists and residents working in The Department of Cardiology for their help in eligibility screening of study candidates and their participation in the enrollment of patients. Moreover, we are grateful to all nurses involved in blood sample collection for their contribution to the study.

\section{Funding}

The study is funded by Collegium Medicum of Nicolaus Copernicus University (NCU CM grant no. 202) and did not receive any external funding.

\section{References}

1. Roffi M, Patrono C, Collet JP et al. 2015 ESC Guidelines for the management of acute coronary syndromes in patients presenting without persistent ST-segment elevation. Eur Heart J 2016; 37: 267-315.

2. Steg G, James SK, Atar D et al. ESC Guidelines for the management of acute myocardial infarction in patients presenting with ST-segment elevation. Eur Heart J 2012; 33: 2569-2619.

3. Wiviott SD, Braunwald E, McCabe $\mathrm{CH}$ et al. Prasugrel versus clopidogrel in patients with acute coronary syndromes. N Engl J Med 2007 357: 2001-2015

4. De Servi S, Goedicke J, Schirmer A, Widimsky P. Clinical outcomes for prasugrel versus clopidogrel in patients with unstable angina or non-ST-elevation myocardial infarction: an analysis from the TRI TON-TIMI 38 trial. Eur Heart J Acute Cardiovasc Care 2014: 3:363-372.

5. Wallentin L, Becker RC, Budaj A et al. Ticagrelor versus clopidogre in patients with acute coronary syndromes. N Engl J Med 2009; 361:1045-1057.

6. Lindholm D, Varenhorst C, Cannon CP et al. Ticagrelor vs. clopidogre in patients with non-ST-elevation acute coronary syndrome with or without revascularization: results from the PLATO trial. Eur Heart $J$ 2014; 35:2083-2093.

7. Navarese EP, Buffon A, Kozinski M et al. A critical overview on ticagrelor in acute coronary syndromes. QJM 2013; 106:105-115

8. Navarese EP, Verdoia M, Schaffer A, et al. Ischaemic and bleeding complications with new, compared to standard, ADP-antagonist regimens in acute coronary syndromes: a meta-analysis of randomized trials. QJM 2011; 104: 561-569.

9. Winter MP Koziński M, Kubica J, Aradi D Siller-Matula JM. Personalized antiplatelet therapy with $\mathrm{P} 2 \mathrm{Y} 12$ receptor inhibitors: benefits and pitfalls. Postępy Kardiol Interwencyjnej 2015; 11: 259-280.
10. Hobl EL, Stimpfl T, Ebner J et al. Morphine decreases clopidogrel concentrations and effects: a randomized, double-blind, placebo-controlled trial. J Am Coll Cardiol, 2014; 63:630-635

11. Kubica J Adamski P Ostrowska $M$ et al. Morphine delays and attenuates ticagrelor exposure and action in patients with myocardial infarction: the randomized, double-blind, placebo-controlled IMPRESSION trial. Eur Heart J 2016; 37: 245-252

12. Kubica J, Adamski P, Ostrowska $M$ et al. Influence of morphine on pharmacokinetics and pharmacodynamics of ticagrelor in patients with acute myocardial infarction (IMPRESSION): study protocol for a randomized controlled trial. Trials 2015; 16: 198

13. Adamski P, Ostrowska M, Sroka WD et al. Does morphine administration affect ticagrelor conversion to its active metabolite in patients with acute myocardial infarction? A sub-analysis of the randomized, double-blind, placebo-controlled IMPRESSION trial. Folia Medica Copernicana 2015; 3: 100-106

14. Hobl EL, Reiter B, Schoergenhofer C et al. Morphine Decreases Ticagrelor Concentrations but not its Antiplatelet Effects: A Randomized Trial in Healthy Volunteers. Eur J Clin Invest 2016; 46: 7-14.

15. Kubica J, Kozinski M, Navarese EP et al. Cangrelor: an emerging therapeutic option for patients with coronary artery disease. Curr Med Res Opin 2014; 30: 813-828.

16. Alexopoulos D, Xanthopoulou I, Gkizas $\vee$ et al. Randomized assessment of ticagrelor versus prasugrel antiplatelet effects in patients with ST-segmentelevation myocardial infarction. Circ Cardiovasc Interv 2012; 5: 797-804

17. Parodi $G$, Valenti $R$, Bellandi $B$ et al. Comparison of prasugrel and ticagrelor loading doses in ST-segment elevation myocardial infarction patients: RAPID (Rapid Activity of Platelet Inhibitor Drugs) primary PCI study. J Am Coll Cardiol 2013; 611: 601-1606.

18. Kozinski M, Pstragowski K, Kubica JM et al. ACS network-based implementation of therapeutic hypothermia for the treatment of comatose out-of-hospital cardiac arrest survivors improves clinical outcomes: the first European experience. Scand J Trauma Resusc Emerg Med 2013; 21: 22.

19. Zafar MU, Farkouh ME, Fuster V, Chesebro JH. Crushed clopidogrel administered via nasogastric tube has faster and greater absorption than oral whole tablets. J Interv Cardiol 2009; 22: 385-389.

20. Parodi G, Xanthopoulou I, Bellandi B et al. Ticagrelor crushed tablets administration in STEMI patients: the MOJITO study. J Am Coll Cardiol 2015; 65: 511-512

21. Moher D. CONSORT: an evolving tool to help improve the quality of reports of randomized controlled trials. Consolidated Standards of Reporting Trials. JAMA 1998; 279: 1489-1491.

22. Rennie D. CONSORT revised - improving the reporting of randomized trials. JAMA 2001; 285: 2006-2007.

23. Kozinski M, Bielis L, Wisniewska-Szmyt $\mathrm{J}$ et al. Diurnal variation in platelet inhibition by clopidogrel. Platelets 2011; 22: 579-587.

24. Koziński M, Bielis L, Wiśniewska-Szmyt $J$ et al. Increased morning ADP-dependent platelet aggregation persists despite dual antiplatelet therapy in patients with first ST-segment elevation myocardial infarction: Preliminary report. Cardiol J 2008; 15: 530-536.

25. Koziński M, Obońska K, Stankowska K et al. Prasugrel overcomes high on-clopidogrel platelet reactivity in the acute phase of acute coronary syndrome and maintains its antiplatelet potency at 30-day follow-up. Cardiol J 2014: 21: 547-556. 